\title{
PRODUCCIÓN Y VALIDACIÓN DEL CONOCIMIENTO CIENTÍFICO
}

Si hoy se puede hablar de una crisis de desarrollo de la filosofía de la ciencia es sobre el trasfondo de una producción riquísima en contenido, elaborada durante cinco decenios en forma esencialmente colectiva y con características que tradicionalmente se atribuían sólo a la investigación científica. Para nada de algo así como una filosofía "blanda". Algunos caracteres de esa crisis aparecerán en la sección V del presente trabajo. En cuanto al trasfondo' no lo consideraremos explícitamente aquí. ${ }^{1}$ No enfocaremos esa crisis én sus aspectos más generales sino a través de una concepción que ha sido dominante y que no lo ha sido como un contenido (o un problema más), ${ }^{2}$ sinó como el marco en que se ha desarrollado, y aislado la disciplina durante un buen período. A ese aislamiento le adscribiremos una función ideológica, es decir de ocultamiento del hecho de que la actividad "reconstruida", la investigación científica, requiere para ser entendida algo más, que no se pone de rélieve y que por el contrario, se excluye. Se consuma aquella tarea por medio de la reconstrucción racional del conocimiento científico, fundamentalmente encarada como análisis de la estructura "final" - o momentánea, corte transversal - y toda otra consideración se efectúa en esa perspectiva a los eféctos de entender esa estructura interna. Ahora bien, la concepción que de ese modo sirve de marco a la actividad epistemológica ${ }^{3}$ deslindándola dé toda otra, es la que se dio en llamar "de los dos contextos"; contexto de descubrimiento y contexto de validación son así los polos, intratable y tratable, respectivamente, que por ello van a tener distinto destino. El segundo elaborado magníficamente y el primero omitido en forma aparentemente fundada. ${ }^{4}$ Sólo que tanto el propio desarrollo de los aspectos epistemológicòs así delimitados, cómo el desarrollo masivo de la producción científica, como la conciencia que ésta generó de que se estaba olvidando algo fundamental, contribuyeron a un cambio profundo que sólo equivocadamente se puede llamar crisis a secas — con todas sus connotaciones catastrofistas - cuando en realidad se tra-

1 Lo hicimos en un artículo anterior ("Situación y funciones actuales de la filosofía de la ciencia", Cuadernos Uruguayos de Filosofia, Montevideo, v. 3, 1964), en algunos de sus aspectos.

2 Los problemas polares de la filosofía de la ciencia han variado en el periodo indicado (ver ejemplificación en la sección 5 ).

3 Se utilizarán indistintamente los términos "epistemología" y "filosofía de la ciencia".

4 Lejos está de nosotros, como se verá, pensar como viables posibilidades del tipo de una "lógica del descubrimiento". 
ta de una ruptura del marco tradicional, de lo que hemos llamado una crisis de desarrollo. Pero lo notable, lo que hace de ella una crisis no cualquiera de ese tipo, es que la elaboración epistemológica por sí parece a todas luces estar mordiendo su propia cola. En efecto, los problemas surgidos respecto al concepto de validación -que ponen totalmente en cuestión la concepción de los dos contextos, es decir el propio marco de la concepción tradicional de la filosofía de la ciencia_ se sitúan de nuevo, como el principio, en el centro de la cosa.

\section{II}

Partimos de Reichenbach (Experience and Prediction, 1938, esp. parágr. 1). Distingue tres tareas para la epistemología [filosofia de la ciencia] que dan lugar a enfoques correlativos: descriptivo, crítico y asesor [counseling task]. La descripción del conocimiento tal como acontece efectivamente hace que la epistemología sea, en ese aspecto, parte de la sociología (p. 3), aunque en un sentido muy particular de este término (en cuanto esa disciplina trate las relaciones internas, el contenido del conocimiento). ${ }^{5}$ La epistemología se distingue por otra parte de la psicología porque "intenta construir los procesos de pensamiento como deberian, suceder si han de ser ordenados en un sistema coherente" (p. 5).$^{6}$ Se trata de reemplazar, en los procesos de pensamiento, los pasos intermedios reales situados entre el punto de partida y el de llegada de aquéllos, por conjuntos de operaciones justificables, por tanto construidos. "La epistemología considera un sustituto lógico más que procesos reales" (p. 5); en ello consiste en lo esencial la reconstrucción racional para Reichenbach. Debe recordarse pues que "nunca será una objeción permisible a una construcción epistemológica que el pensamiento efectivo no se conforme a ella" (p. 6); sin embargo nos dice asimismo que la descripción, que no es copia es de todos modos un equivalente y está sujeta al postulado de correspondencia (p. 8). El concepto de reconstrucción racional "corresponde a la forma en que los procesos de pensamiento son comunicados a otros en lugar de la forma en que son subjetivamente realizados" (p. 6). Publicación (presentación pública) y búsqueda ( $\mathrm{y}$ hallazgo). pertenecen a contextos diferentes, respectivamente de justificación y de descubrimiento. La tarea descriptiva, en ese sentido, de la epistemología da lugar a la reconstrucción racional cuyo ejemplo sólo aproximativo se da en la comunicación.

Sin embargo no hay para Reichenbach un corte totalmente definido entre esta tarea y la crítica, ejerciendo el concepto de reconstrucción racional

5 No parece totalmente justificado utilizar el término "sociologfa" de este modo. Reichenbach no es suficientemente claro en sus ejemplos; aparte de sus deslizamientos entre "descripción" y "construcción". Con respecto al uso de la expresión "reconstrucción racional", dentro de las limitaciones señaladas, debe decirse que se aplica' en Experience and prediction a modelos generales y no a reconstrucciones específicas de teorias particulares.

B. Subrayado nuestro. 
el papel de puente entre ambas. De todos modos la crítica se realiza para determinar la validez y la confiabilidad del sistema de conocimiento. Ya la selección de la reconstrucción racional está guiada, en la descripción, más allá de su tentada "correspondencia" con procesos efectivos, por su : justificabilidad. Las operaciones sustitutas construidas son tales que puedan demostrarse como válidas, aunque en la faz descriptiva puedan quedar cadenas residuales de pasos no justificables. La tarea crítica se sitúa por eso en el centro mismo de la epistemología y su resultado es un análisis de la ciencia, una lógica de la ciencia.

De todos modos existen además pasos científicos no guiados por un principio de validez sino sujetos a decisiones voluntarias. "Que hay ciertos elementos del conocimiento sin embargo, que no están gobernados por la idea de verdad sino que están sujetos a decisiones voluntarias y que aunque influyen de modo acentuado la construcción del sistema total de conocimiento, no alteran su carácter de verdadero, es algo menos conocido por los investigadores filosóficos" (p. 8). Las convenciones (por ejemplo: tanto de unidades de medida; como acerca de ciertas características básicas del espacio-tiempo), aunque son fruto de decisiones dan lugar a teorías equivalentes; ${ }^{7}$ en cambio otras decisiones (por ejemplo postulados sobre los objetivos de la ciencia) dan lugar a bifurcaciones [volitional bifurcations] y por tanto a teorias sensiblemente distintas. Reichenbach se refiere brevemente al status de las decisiones y de los enunciados que se refieren a ellas. ${ }^{8}$ El concepto de decisión abre una tercer tarea a la epistemología, de asesoramiento [advisory task], que en esa función efectúa propuestas [proposals]. Un concepto importante a ese respecto es el de decisiones encadenadas [entailed decissions] pues permite en algunos casos volver de esta tercer epistemología a la segunda, del modo siguiente: "renunciando a hacer una propuesta y construyendo en su lugar una lista de todas las decisiones posibles con las que están encadenadas cada una de ellas" (p. 14). Por otra parte Reichenbach ve en el concepto de decisiones encadenadas la forma de 1) escapar al convencionalismo (arbitrariedad derivada de las decisiones adoptadas) y 2) separar partes arbitrarias y sustantivas, subjetivas y objetivas, respectivamente, de la ciencia. Parece en cambio menos convincente el complemento que propone a través de la noción de aceptación universal de ciertas decisiones, como base de las'cadenas decisionales.

A manera de resumen diremos que:

7 No discutiremos este punto.

8 Las decisiones, como es obvio, no poseen valor veritativo. Existen dos tipos de enunciados interesantes acerca de ellas: 1) los que dicen qué decisiones utiliza la ciencia en su práctica (descripciones sociológicas), y 2) los que dicen que se da una decisión y no un enunciado (epistemología crítica sobre un hecho lógico). Una decisión no es un hecho pero su carácter de ser una decisión es un hecho y puede aparecer en un enunciado (Experience and prediction, $\mathrm{p}: 11$ ). 
1. No consideraremos algunas confusiones y deslizamientos apuntados apenas al pasar - en algunos casos- en el texto de Reichenbach. 2. Aunque la tradición recogió en general la distinción entre contextos -y la de recons. trucción racional, explícitamente formulada - lo hizo rápidamente, tomándola como aproblemática, e interpretándola de manera algo variada. Probablemente ello se deba a que Reichenbach la introduce por medio de ejemplos, insuficientes. 3. Reichenbach propone tres retornos a la epistemología crítica -terreno más firme si los hay- a partir de la región "conflictual" de las decisiones, pero la tradición, que mantuvo sobre todo la idea de distinción entre contextos, no prestó mayor atención a este aspecto más complejo. y significativo. Es un mérițo de Reichenbach haber insistido en él. Los tres retornos son: a) a través de la aceptación universal de decisiones básicas - de dudosa aceptación universal - , b) a través de las cadenas decisionales presentadas a modo de opciones - completas- entre decisiones alternativas, y c) diciéndonos al pasar - sin mayor fundamentación_ que aquellas decisiones "no alteran su carácter de verdadero [el carácter del sistema total de conocimiento]". (p. 8). Hay por lo tanto problemas ricos planteados junto a hilos sueltos; no fueron ni recogidos los primeros ni atados los segundos.

\section{III}

Los postulados que conducen a bifurcaciones teóricas parecen más abundantes y profundos en sus consecuencias que los solos referentes a los objetivos de la ciencia (ejemplo principal presentado por Reichenbach).

El enraizamiento en tradición intelectual de la distinción entre contextos nos interesa por su influencia manifiesta y por sủs consecuencias. $\mathrm{Y}$ el hecho de que no se trabajara mayormente la idea de decisiones encadenadas (más allá de si el "retorno" propuesto por Reichenbach y su "base" son lícitos), aunque apuntara a un tratamiento formal pertinente a aspectos menos formales, parece una constatación más de que la pragmática de la ciencia, por deseable que fuera, no se desarrolló a partir de su programa, por otra parte no siempre preciso.

Presentar las vicisitudes que sufrió posteriormente la distinción tajante entre contextos (por lo demás bastante modestas). no es lo que nos interesa aquí. De todos modos, para tomar sólo un ejemplo, el volumen quinto de los Minessota Studies in the Philosophy of Science (1970), al plantear las relaciones entre historia y filosofía de la ciencia a través de distintos aportes, supone en casos especificos algunos de los problemas de la distinción, lo que no es para nada casual dado el momento en que aparece.

En especial W. Salmon trata'explícitamente el tema (vol. cit., "Bayes's theorem and the history of science", p. 68-86). Frente a quienes sostienen que "el estudio de la ciencia conclusa no es para nada el estudio de la cien- 
cia", que "no se puede entender la ciencia a menos que se vea cómo crece" y que "para comprender la estructura de la ciencia es necesario dar cuenta del cambio y de la revolución científica" (p. 69). Salmon se confiesa un "irre; constructo reconstruccionista" y afirma que "la distinción es viable, significante y fundamental para la filosofía de la ciencia". (p. 7o). Pero, a su vez, sin alterar lo esencial, trata de limar'los obstáculos con que tropiezan algunas presentaciones de la distinción. 'En especial muestra cómo los dos contextos tienen items en común al punto de que "el proceso de descubrimiento y: el de justificación pueden' ser cas̀i idénticos" (p. 72): Para él "no hay razones por las cuales uno y el mismo item no pueda ser a la vez psicológica y lógicamente relevànte a una hipótesiśs dada" (p. 7o)." Pero concluye: "Lós contextos se contrastan entre si, no sobre la base de que no puedan tener miembros en común sino sobre la base de las diferencias en los tipos de relaciones que incorporan" (p. 7o). De todos modos su tratamiento anexo del contexto de justificación, que de lograr resultados probaría aquella: viabilidad concreta, se realiza (hacia el fin de; su trabajo) sobre la base del teorema de Bayes's y comporta una-reconstrucción inductiva del conocimiento que presenta inconvenientes" serios que no corresponde tratar aquí.

Bajo el término "descubrimiento" y bajo la expresión "contexto de descubrimiento" caen variados elementos, lo que permitió utilizar abundantemente la distinción en dos contextos sin que sirviera para mucho teóricamente.10

De aquí en adelante distinguiremos en el llamado contexto de descubrimiento: a) los procesos subjetivos ${ }^{11}$ que llevan a obtención de conocimientos, y b) todos los demás aspectos de la producción de conocimientos (reservaremos de, aquí en adelante el término "producción" para designarlos). Se trata en lo esencial en a) de aconteceres individuales que caen dentro del ámbito de la psicología: ${ }^{12}$.

Las propuestas de "lógicas" del descubrimiento (de Peirce a Hanson por 10 menos) fueron desechadas sistemáticamente y no sin fundamento. Los aspectos psicológicos se mostraron especialmente reacios al establecimiento de legalidades y eso dio la tónica para que todo el contexto de descubrimiento fuera más o menos intratable (el descubrimiento científico y la creación artística aparecieron como procesos nada lejanos en este sentido).

Dé cualquier manera pareció sumamente difícil que, el amplio tema del descubrimiento pudiera a la vez adaptarse a contener, las exquisiteces de la creación como acto individual con sus condiciones específicas (con todas las variantes que llenarọn, el rico anecdotario de una historia ${ }_{\text { evenementielle }}$

9 "Viable" es poco decir.

10. Lo cual no quiere decir que no haya sido utilizada, como dijimos, profusamente.

11 Consideramos que este término es suficientemente claro, aunque podría estar sujeto a mayor elucidación.

12 En el sentido de la obra de Hadamard sobre la invención matemática. 
-hechista - de la ciencia), en un extremo, y ajustarse además a los esquèmas fórmales bien establecidos, aún en su variedad, que reciben adecuadamente el nombre de lógica (deductiva). ${ }^{13}$

La tendencia corriente fue encarar la reconstrucción racional de los sistemas científicos prescindiendo de todos los problemas llamados de génesis de modo que una enorme caja negra contuvo, para la filosofía ortodoxa de lä ciencia, un cúmulo de elementos muy variados. Aquí se propone utilizar en cambio una caja negra mucho más reducida y'mantener como tarea el volver traslúcidos, como se ha 'dicho, o quizás transparentes, los "envases" de la producción de conocimientos. Se han dado razones a nuestro entender suficientes, y: que no vamos a explicitar aquí, para mostrar la relativa independencia ${ }^{14}$ del tratamiento de $b$ ) respecto al de a) (hipótesis 1.). De modo que tendríamos momentáneamente tres contextos: de descubrimiento - sensu stricto-, de producción, y de validación. ${ }^{15}$ El estudio del segundo de ellos es por su parte asunto:complejo que pertenece a niveles también variados pero suficientemente conectados para tener por ${ }^{1}$ el momento un foco común. Abarca estudios empíricos y otros que no lo son tanto, investigaciones que han tenido lugar - con detalle o no- en los últimos decenios, y corazonadas [hunches] que apenas intentan: por ahora sêr algo más que tales. De todos modos nos interesa demostrar cómo el hiato que se estableció entre los dos contextos de Reichenbach debe ceder su lugar a relaciones mucho más matizadas entre producción y validación.

Nos interesará señalar brevemente el uso ideológico que se ha hecho de la distinción entre los dos contextos originales, y previo a ello, mostrar no tanto que toda distinción se borra sino que la importancia creciente del contexto de producción aconseja asumir una posición cauta respecto a la actitud corriente de considerar la validación en forma totalmente independiente y por tanto autosuficiente. Por ello a nuestro entender la filosofía de la ciencia de raíz neopositivista (y corrientes emparentadas) aun si abandonó tesis fundamentales, con la dominancia que ha tenido - -y justamente por, ella-, se ha hecho culpáble a veces, : y cómplice otras, del desconocimiento de aspectos fundamentales de la producción de conocimientos. Es más, pensamos que un proceso muy especial que se ha dado en el desarrollo del conocimiento científico en los últimos decenios ha descolocado los planteos de aquella raíz; no es extraño pues que los aspectos "ecológicos", a la vez imprescindibles y de

13 No cuenta como tal, como es obvio, la llamada lógica indùctiva:

14 En el sentido en que Braithwaite (Scientific Explanation) considera el tema de la explicación como independiente del de la naturaleza de la base empirica.

15 Por razones que vamos a ver más adelante (sección 5 a) parece razonable utilizar en lo sucesivo, desde ya, el término "validación" en lugar del término "justificación" de Reichenbach. Aunque podrían utilizarse también algunos otros (p. ej. comprobación) tomamos el que nos propone Ferrater en su Cambio de Marcha en Filosofia. Preferimos, en ese sentido, reservar "justificación" para un uso diferente. 
moda; deban adquirir importancia en filosofía de lá ciencia visto el medio ambiente cambiante de la investigación científica y el aporte creciente del terreno que la nutre.

$\therefore$

\section{IV}

Con toda la arbitrariedad que los hitos cronológicos poseen, es necesario reconocer de algún modo los cambios de la cualidad que no dejan de hacerse presentes aún en la investigación científica.

A partir de la última "guerra mundial" y especialmente de la confrontación que tiene lugar a partir de ella, la investigación científica adquiere ciertos caracteres distintivos. No se trata de una aparición súbita sino de cambios graduales que por su volumen constituyen un fenómeno nuevo (hipótesis .2.). Là producción dirigida a los fines de la guerra hace surgir -en el período 1939-45- una orientación neta de la investigación (pensemos en èl papel de E. Snow en Inglaterra pero no se trata de un caso aislado sino sólo de un ejemplo repetible; quizás más impresionante sea el proyecto "Los Alamos"). Pero ese proceso de corta duración y con fines precisos de desarrollo de tecnologías ya bastante finas y complejas (diferentes al uso de tanques o de la aviación en 1914-18) se consolida y amplifica en el marco de la guerra fría y de la contienda llamada ideológica (por momentos más que tibia) que conducen a dos series de acontecimientos sólo tenuemente desvinculados, la carrera nuclear y la carrera espacial. Si la economía de los paises en guerra se orientó por los fines de ésta, más aún ambas carreras dirigieron, en el período posterior, el total de las economías de los países desarrollados, a lós fines de la confrontación. A tal punto que las vicisitudes de esa pugna incidieron decisivamente en la situación de empleo y de "salud" de la economía (pensemos en la inflexión que se da a partir del momento en que se llega a la Luna; la NASA y las empresas correlacionadas'a ese esfuerzo determinan el empleo no sólo de personal altamente calificado sino de toda clase de empleo aúxiliar, en la puesta a punto de artefactós tecnológicos finos y también en los subproductos de la investigación y tecnología, e inciden decisivamente en el aumento de la tasa de desempleo y en las cifras globales, críticas más allá de determinado umbral, desde el momento en que se cumplieron las metas. fijadas). ${ }^{16}$

Pero sería erróneo pensar que las carreras nuclear y espacial son las únicas que viven este proceso, más allá de que su volumen suministra los datos más notorios. El conjunto de la investigación científica, aún de la aparentemente desvinculada dé aquéllas, se orienta por pautas bien definidas. Los

$16^{\prime}$ Es cierto que la crisis del petróleo incidió complèmentariamente en la salud de la economía y por tanto en la situaciớn de desempleo. No sólo la dinamicidad del empleo de cientfficos disminuyó sino que aún las plazas dé filosofía de la ciencia estuvieron sujetas al mismo fenómeno, por lo menos en Estados Unidos. 
organismos de financiación (no sería necesario nombrarlos; bastan los ejemplos de la National Science Foundation y de las fundaciones "privadas") determinan qué se hace, de modo que la investigación individual con metas propias - y éstas han sido poco estudiadas globalmente en cuanto a sus determinantes implícitas - pasa a ser un fenómeno relativamente folklórico (estadísticamente considerada). De todos modos el volumen de la investiga-. ción crece de modo exponencial (aún teniendo en cuenta las inflaciones señaladas) al punto que se la cuantifica ya en la forma de F. Machlup. (en su Production and distribution of knowledge in the United States, 1962). Pero el volumen de la investigación no crece de cualquier modo sino hacia la obtención de metas bien definidas en la aplicación del conocimiento y éstas no se logran sino a través de una planificación cuidadosa; se lás financia por tanto selectivamente. Por ello (y aquí sólo recordamos hechos conocidos y señalados en general pero. no suficientemente estudiados en su detalle; sin embargo una creciente literatura los enfoca), el volumen, la dirección aplicada - aún de la investigación "básica"-, la planificación y: por'consiguiente la financiación masiva, son fenómenos que determinạ un salto de cualidad en la investigación científica. El conjunto de esos elementos se engloba en un mismo rubro que enfoca la política cientifica. Lo que interesa aquí es la determinación de las líneas y resultados (buscados) de investigación, por la política científica de los países desarrollados. Pero no debe pensarse que ello. se da solamente en el marco de éstos. No sólo también las potencias secundarias actúan de modo coherente con el señalado (pensemos en la pólítica de investigación y de producción nucleares en potencias de segundo orden como Francia, India, Israel o Sudáfrica) sino que se presentan aún en la creación de organismos de planificación (económica y científica) en el llamado Tercer Mundo. ${ }^{17}$ Los gobiernos que pretenden seguir líneas de independencia condicionan su financiación de la investigación a fines nacionales concre tos (ello aparte de las determinaciones de la investigación efectuadas fuera de esa planificación nacional y proveniente sea de fundaciones nacionales o internacionales, ${ }^{18}$ sea por circunstancias de prestigio ${ }^{18} \mathrm{u}$ otras -autolimitación de los científicos a temas de interés nacional).

it Es el ejemplo del oonacyt en México.

$18 \mathrm{La}$ concentración durante cierto periodo en Argentina en investigaciones acerca de las 'particulas elementales, con la infraestructura cara y rápidamente superada, que exigen, es un ejemplo de la influencia muchas veces nefasta de pretender estar "en. la punta" en temas de prestigio. La influenciá de la "ayuda extranjera" hizo derivar en Uruguay hacia estudios de fisiología de la retina o del parto "más allá de su importancia intrínseca, por. sus fuentes de financiación) que intentaron , resolver problemas ajenos (de la Fuerza Aérea de los Estados Unidos en un caso, de problemas perinatológicos en la perspectiva y necesidades de ese pais, en el otro). El caso del estudio en -Uruguay de la plaga que constituyen los camalotes en vías fluviales tenía áplicación a la desembocadura del Mississippi, y para:

I9 Publicar artículos en revistas cotizadas internacionalmente pasó a ser un determinante frecuente en ese sentido. . 
Las politicas cientificas, independientes o no; explicitas o no, determi. nan por doquier el curso de la investigación, aplicada o no. De modo que toda ${ }^{*}$ otra investigación ha adquirido de hecho un carácter marginal. Más allá de que se hable de libertad de investigación, ésta está:sujeta a un estrechísimo marco, y no es difícil' pensar que cada vez lo estará 'en mayor grado. Por ello esa marginalidad creciente, sin perjuicio de su importancia individual, no es un fenómeno más sino una característica central que debe tomarse en cuenta en todo análisis teórico del desarrollo de la investigación hoy, y de cada uno de sus momentos. Esa dominancia, y' esá marginalidad correlativa (hipótesis 3) no son por ello nada triviales sino tódo lo contrario. Cada proceso de investigación es acompañado además por la evaluación - cieñtífica y no- de sus resultados que realimenta el proceso de planificación. Por todo esto las condiciones dé producción adquieren un significado epistemológico más allá del que poseían de por sí.

Es obvio que esas constataciones conllevan un cambio en la manera de realizar estudios metacientíficos (y en especial de filosofía de la ciencia).

No es sin fundamento suponer que el propio desarrollo de la filosofía de la ciencia ha experimentado cambios correlativos (hipótèsis ' $2^{\prime}$ ) que han sido todavía poco éstudiados y qüe los seguirá experimentando.

Pero lo que nos interasa más aquí es señalar cómo el contex́xto de producción pasa a ser determinante (parả nada descartable, o archivable, en lo que originariamente recibió el nombre de contexto de descubrimiento, como tarea a su vez marginal), y cómo la importancia de su intervención atenúa de modo significativo el aislamiento de la considéración en el análisis dé la validación, instancia excluyente y aún definitoria de la profesión "del filósofo de la ciencia." Baste ello para justificar la "disgresión.

\section{$\mathbf{V}$}

La reconstrucción racional de los sistemas científicos ha sido de una mañera u otra el tema de la filosofía de la ciencia en los últimos cuarenta años. Cuando digo "de una manera ư otra", apunto al hecho de que, más allá de la variedad de las teorías y de los problemas que cada línea de investigación, y aun tendencia, se ha planteado, el curso de la investigación ha dado lugar a una proliferación difícil de seguir y por ende de entender.

Bastaría recorrer sólo la colección de Philosophy of Science durante los quince o veinte últimos años para percibir una doble maraña creciente en los intentos de reconstrucción racional (y ello se acentúa a medida que consideramos periodos más cortos y recientes): Valga como ejemplo la discusión

nada a là situación local. Mas allá de que en Uruguay hubiera una fuerte resistencia a la "ayuda externa" por los condicionamientos de todo tipo que involucra; esos ejemplos _que son excepciones dentro de una política en general adecuada y puesta realmente en práctica-; muestran de todos modos la trama de esa "ayuda". 
cada vez más detallada y laberíntica del modelo nomológico-deductivo de Hempel-Oppenheim y de sus implícitos.

Digo doble maraña para distinguir dos tipos de elaboraciones: I) las que toman como centrales uno $\mathrm{u}$ otro de temas tales como criterios de significación a de demarcación, estructuras de la explicación (y de la predicción), grados de confirmación de hipótesis, status de los términos teóricos, comprobabilidad global de las teorías (versus comprobabilidad de enunciados rèlativamente aislados), para nombrar apenas algunos de los que atrajeron la atención, y 2) elaboraciones de detalle, dentro de cada uno de esos enfoques; de términos tales como grue u otros. Todo el proceso de pensamiento a que nos referimos - sin que los ejemplos recordados puedan clasificarse rigurosamente àsí (nos interesa sólo mostrar su extremada complejidad en niveles distintos) - es, a través de su por lo menos doble variedad; un intento significativo de producir una reconstrucción racional adecuada del conocimiento científico. Pero el hecho es que, más allá de que una u otra concepción (o tema) fuera dominante durante ciertos periodos (como lo fue por ejemplo ampliamente la de entender los sistemas científicos como sistemas interpre:tados con reglas de designación), lo cierto es que no se ha producido una reconstrucción racional medianamente aceptable.

A este respecto y para "sustituir" una historia y evaluación detalladas fuera de lugar aquí, quizás como argumento de autoridad, con todas las limitaciones que estos encierran, podríamos recordar las frases finales de la Philosophie de la Physique de M. Bunge (quien luego de presentar un modelo complejo de reconstrucción racional), dice: "En la medida en que se acepta lo que acabamos de decir [la teoría y la experiencia no se encuentran jamás en un combate singular; su encuentro se sitúa en un nivel intermedio, en presencia de elementos teóricos y empíricos agregados, en presencia en particular de los modelos teóricos que describen a la vez el objeto de la teoría y el dispositivo experimental empleado], se debe considerar que la filosofía de las ciencias dominante a la hora actual es inadecuada. Es necesario volver a comenzar desde cero, buscando no separarse de la práctica real de la investigación cientifica, aun a costa de abandonar ciertas tradiciones filosóficas" (op. cit., p. 294, subrayados nuestros).

Sin embargo, para eliminar ese carácter de autoridad, asumimos lo que se afirma allí como hipótesis de trabajo (hipótesis 4 ), sujeta a que se aporte separadamente a su respecto mayores elementos de prueba que los que se ha suministrado.

Esta que parece ser la situación real - cúmúlo de teorías y sub-teorfas epistemológicas y falta de decisión visible- constituye por eso en lo esencial una inadecuación en la provisión de una reconstrucción racional aceptable.

Ahora bien, si la distinción entre lós dos contextos suponía una reconstrucción racional que de algún modo fundara su aplicación, podemos razo- 
nar, provisoriamente, de este modo: $x$ ) mientras tal reconstrúcción no exista la distinción no es sostenible; 2) tal reconstrucción racional adecuada no existe; 3) luego...

$\therefore \quad$ No obstante interesa considerar algünos elementos complementarios; no ya para matar un caballó muierto más sino para intentar evaluar por lo menos parcialmente la situación de los contextos epistemológicamente necesarios, y en todo cáso, pára: proponer una solución sustitutivá (y enfocar los̀ problemas específicos que ésta impone), que cumpla una función no ideológica y que sea en ese sentido, también, más fértil.

Ahora bien; para circunscribir el tema; aparte de ciertas posturas extre: mas ${ }^{20}$ dentro de las reconstruciones que atribuyen al concèpto de teoría un papel central, "podemos distinguir, siguiendo provisoriamente la concepción tipo "pastel de ‘capas". [layer-cake theories]; $x$.) un conjunto de enunciados de distintos niveles que poseen valor veritativo ${ }^{21}$ frente a 2 ) otros que no lo poseen pero que intervienen de todos modos en general explícitamente en la construcción : de teorías científicas. Por otra parte debe reconocerse también la participación en la empresa cientifica de 3). un conjuntó de presupuestos que, en la mayor parte de los casos se dan en forma implícita, pero no menos actuante. En qué medida los dos últimos tipos señalados intervienen en' la estructuración misma (en sus cortes transversos) y son por ello dignos de formar parte de la reconstrucción racional deseada, es un problema abierto: Pero justamente la distinción del contexto de validación o sus alternativas supone considerar de modo preferente esa intervención (por sus consecuencias).

a. En primer lugar consideraremos esos elementos: que intervienen en la construción de teorías sin poseer por sí mismos valor veritativo y que de todos modos condicionan el valor veritativo de éstas (por lo 'menos en algunos casos) y especialmente el curso de la empresa científica en conjuntó en cuanto ella intenta de algún modo reflejar adecuadamente el mundo. Es dudoso que esos elementos puedan sè confinados a una consideración de la sola problemática, o en todo caso lo que está en cuestión es si puede aislarse a ésta de las otras formas de consideración.

3 Me refiero en especial a las prescripciones metodológicas y a los. enunciados ${ }^{22}$. nomopragmáticos modales '(tipos de enunciados nomopragmáticos en

20 El caso de Feyerabend con su anarquismo epistemologico, sin perjuicio de sus criticas válidas a elaboraciones a veces vacias, es un ejemplo de esas posturas extremas.

21. Más allá del problema de la adscripción de valores veritativos a las hipótesis universales, por ejemplo, que en principio lo poseen, distinguimos los casos de instancias en que ni aún' en esè sentido puede hablarse de valores veritativos (es claramente el de las instancias decisionales). -

.22 Bunge usa "enunciado" con un sentido general, aun si se trata de aquellos que no poseen valor véritativo." No hemos encontrado en la literatura un términö adecuado para este uso. 
el sentido de M. Bunge, The Myth of Simplicity, caps, 10 y 12), a los enunciados metanomológicos normativos y a los enunciados metanomológicos metodológicos (también siguiendo la clasificación presentada en dicha obra). Habría que preguntarse si todos esos enunciados ${ }^{23}$ pertenecen estrictamente al corpus de la ciencia y si son por tanto dignos de participar en su reconstrucción racional. Es claro que los enunciados no figuran en las reconstrucciones racionales corrientes pero son éstas las que están en cuestión. Pero además puede decirse que bastaría que uno solo de esos tipos de "enunciados" (o en especial las propuestas [proposals], ciertas convenciones por ejemplo, ciertos momentos de decisión; y no me refiero para nada a los enunciados que pertenecen a ciencias "anteriores" a la dada) se mostrara como imprescindible (hipótesis 5 ) en la construcción científica conclusa, para que los demás problemas se agravaran con éste en la delimitación del contexto de validación. Al conjunto de propuestas y similares "enunciados" que intervienen, en general explícitamente, de ese modo lo llamaremos $p_{1}$. Determinan indudablemente, por lo menos, el curso de la investigación ${ }^{24}$ pero quizás pudiera objetarse que su consideración pertenece más bien al estudio de la estructura de desarrollo del conocimiento científico que a la reconstrucción racional como corte transversal.

En todo caso su presencia pone en cuestión justamente la separabilidad de ambos tipos de estudio, que era un supuesto de la concepción aquí criticada. Pero aun sin este cuestionamiento, innecesario por el momento, la presencia de $p_{1}$ debe ser reivindicada aun en la presentación de la teoría por más que no sea algo corriente.

Su presencia, y su aceptación fundada, apunta al problema de su justificación (por eso hemos usado "validación" para lo que Reichenbach llama "justificación", reservando este término justamente para encarar este problema). Se ha dicho que la justificación de los enunciados de tipo $p_{1}$ tiene que ver con su fertilidad y con su coherencia respecto al perfecto conocimiento disponible (Bunge, The myth...). Ahora bien la admisión de "fertilidad" o conceptos emparentados, como base para la justificación de esos enunciados, es otra cara del problema que hemos planteado y de la inexhaustividad del concepto de validación.

$b$ : Pero existe además un conjunto, que llamaremos $p_{0}$, de presupuestos en general implícitos, que también condicionan el resultado de la investigación científica (aunque no participen patentemente en ella) y que han sido reiteradamente señalados en la literatura, aunque debe reconocerse que aparecen más frecuentemente en consideraciones "amplias" de la temática científica

23 Distinto al problema de si son leyes, cosa que Bunge, con razón, decide negativamente.

24 Ciencias divergentes en el sentido de Reichenbach: 
que en las formulaciones de reconstrucción racional o en el entorno de éstas. Se trata de presupuestos ontológicos, gnoseológicos, semánticos, lógicos, matemáticos, instituciónales (a ellos nos hemos referido ya), ideológicos, económicos, entre otros, no siempre tan generales o vagos como a menudo se supone. Su consideración puede hacerse más precisa ${ } \mathrm{y} / \mathrm{o}$ perteneciente a las ciencias "anteriores" o puede estar incluida en discursos no científicos, unos suficientemente precisos, otros muchísimo menos; pero su eficacia está de todos modos presente.

Podría de todos modos pensarse, aun en los ejemplos clásicos de intervención de esos presupuestos, que podrían ser eliminables de toda reconstrucción racional adecuada, y confinados a otro tipo de consideración. Lo dudamos. Nuestra hipótesis (hipótesis $5^{\prime}$ ) en 'este caso es que no son totalmente excluíbles de una reconstrucción racional adecuada. Algunas reconstrucciones ya efectuadas, fenomenalistas y fisicalistas, los ponen en evidencia en su propio interior. Pero además la decisión de abandonar nuestra hipótesis supondría de todos modos $I$ ) suministrar una reconstrucción racional adecua: da que no existe (hipótesis 4) y 2) que ella los excluya totalmente. Sin cum: plirse 1) es obvio que 2) no es decidible.

El conjunto de $p_{0}$ y $p_{1}$ por su parte hace dudosa, pues, toda reconstruc: ción racional que los excluya, lo que pone en cuestión la separación de un contexto de validación como instancia excluyente. Pero además muestra cómo el seno de los cortes transversales se ve invadido por las condiciones de producción del conocimiento de un modo difícil de eliminar como otrá instancia interpretatoria.

$\therefore$ Otro aspecto interesante, que sólo se enunciará aqui, es si, y en qué medida, la política científica afecta directamente a los conjuntos $p_{0}$ y $p_{1}$. Una hipótesis plausible (hipótesis 6) es que sí los afecta de modo marcàdo (por lo menos en algunos casos no eliminables).

c. La atención en filosofía de la ciencia ha estado concentrada, sin embargo, en los enunciados con valor veritativo [enunciados sensu stricto]; : en torno a éstos se ha dado lo sustancial de una intrincada producción epistemológica. Hemos señalado antes algunos de los problemas más interesantes y más trabajados, y cómo las respuestas han llevado de todos modos, aun excluyendo los conjuntos $p_{0}$ y $p_{1}$, a una situación totalmente insatisfactoria pese al notorio (pero insuficiente) progreso en las elaboraciones presentadas.

La validación como tal alude a las formas de comprobación de las teorías científicas y de los enunciados que las componen. Para considerar apenas un aspecto, han habido dos modos extremos de concebir la relación entre los conocimientos científicos y los procesos fácticos que los validan. Para un tipo de doctrinas se distinguen por lo menos cuatro niveles de enunciados que interesa justamente validar: $x$ ) los de nivel ínfimo (enunciados particulares 
de observación, interpretados de diversas maneras); 2) las hipótesis de menor nivel (enunciados universales, que incluyen directamente los casos particulares), o estadísticos, según los casos (que presentan problemas"muy especiales, graves por la amplísima participación de esos enunciados en el conocimiento científico); 3) las hipótesis de niveles medios (que contienen indudablemente conceptos teóricos) $\mathrm{y}^{\prime}$ 4) los axiomas (hipótesis de máximo " nivel de las teorias). Respecto a cada üno de los niveles se plantea la cuestión de la validación de sus enunciados. Los problemas que surgen así pueden ser de naturaleza variada: para la llamada baśe empírica por ejemplo se plantea el de su naturaleza y ateoricidad. Para cada uno de los demás se plantean problemas específicos; centrados en la validación misma en el caso de las hipótesis de menor nivel, y en el de los conceptos teóricos para los niveles superiores (como tema intermediario, pero imprescindible, para el de la validación). A medida que se asciende en las capas, el problema de la validación, ya complejo en las inferiores, se hace menos manejable.

En el otro extremo se dan las doctrinas ultraduheimianas, en que nó tiene sentido preguntarse por la validación de enunciados aislados, y que, sin plantear problemas intermedios, remiten el valor veritativo "al todo de la ciencia" (caso notorio de W. Quine en su famoso "Dos dogmas del-empirismo" con su subdeterminación del campo total de los enunciados por la experiencia):

Entre ambos extremos tomamos solamente dos casos. Soluciones produheimianas plantean el problema en términos más restringidos considerando limitadạmente el conjunto $[\mathrm{H}, \mathrm{A}, \mathrm{E}]$ (A, hipótesis auxiliares), a través del modelo nomológico-deductivo modificado (es el caso de los planteos trabajados por A: Grünbaum). Para no nombrar sino un caso más, aparece, indudablemente más próxima a la práctica científica, la doctrina según la cual la validación se da a través de la interconexión, el tejido, de hipótesis y teorías (sin remitir a la dudosa tesis ultraduheimista ya citada) de modo que para ella la validación es un proceso del sistema mismo a través de subsectores. Pero los problemas que se plantea internamente este tipo de posiciones están lejanos de podèrse considerar solucionados.

De modo que, sea un caśo $u$ otro, en el seno mismo de doctrinas epistemológicas que prescinden para la validación de los conjuntos $p_{0}$ y $p_{1}$ (y adhiriéndonos momentáneamente a esa para nosotros infundable reducción) faltan elementos para lograr una reconstrúcción reducida adecuada (hipótesis 7). Por tanto ni aun en ese caso poseemos conceptos de validación de enunciados, o de conjuntos de ellos (teorias), de todos los niveles admitidos por cada doctrina epistemológica. Entonces hemos de concluir que no sólo falta tener en cuenta los elementos de la producción de conocimientos señalados (política científica como dominante, $p_{0}$ y $p_{1}$ ) para considerar la validación de modo 
adecuado sino que; además, es en el propio seno de las propuestas epistemológicas "clásicas", que tenemos una profunda carencia. :

En este caso, pues, no estaría probada para "nada, sino muy lejos de ello, la tesis de una separación abismal entrè el contexto de validación y el de producción de conocimientos (separado éste del contexto de descubrimiento. sensu stricto (hipótesis 1), y menos aún las posibles consecuencias que la to men como fundamento. Es decir que no sólo ya desde fuera sino desde dentro de la producción de reconstrucciones racionales reducidas, aparece, cómo infundado el hiato dominante en la filosofía de la ciencia de los últimos casi cuarenta años.

Un elemento más a tener en cuenta que no consideraremos aqui - pues el concepto de validación sigue para nosotros teniendo -sentido (programático)- - , es el del necesario equilibrio (hipótesis 8) que se da en las teorías cièntíficas entre los elementos de tipo $p_{0}$ y $p_{1}$ por un lado y los "clásicos" por otro, que hace que ellas escapen a las fatalidades del convencionalismo y por ello a un relativismo sin fronteras. De otro modo de ninguna manera las teorias científicas podrían de algún modo reflejar progresivamente el mun: do (hipótesis 9), cosá que nos parece de hecho indudable al nivel de là práctica científica, por más que ese concepto de equilibrio (u otro sustitutivo) no esté elucidado adecuadamente.

$d$. En los años sesenta irrumpe en forma pujante un modo complementario de filosofía de la ciència a través de modelos de la estructura del deśarrollo científico, es decir una visión diacrónica" correlativa a la sincrónica "clásica"." La obra de Kuhn abre ${ }^{25}$ este camino entre las de amplia difusión. "Lo péculiar de ella es proponer hipótesis que pueden funcionar a la vez I) como hilo conductor de una historia de las ciencias antes prácticamente "ateórica" (por lo menos en forma expresa, y 2) como apertura de la filósofía de la ciencia a partir de su dóminancia instantaneísta. Estamos lejos de pensar que el de Kuhn sea un intento logrado, pero es indudable que su discusión ha generado una línea de trabajo descuidada antes sin razón, floreciente a tal punto que difícilmente puede persistir hoy un encerramiento en los temas clásicos.

Con todo no están bien claras las razones de este cambio significativo: (gradual y para nada sustitución): en la producción de filosofía de la ciencia en la última década. Pensamos que ello no es ajeno al cambio en lós condicionantes y volumen de la producción científica misma-(hipótesis 2) pero. decir esto es prácticamente no decir nada si no aludimos a los mecanismos de esa posible relación. Por otra parte el modelo de Kuhn (y sus sucesores. o semi-discrepantes) requiere ábiertamente, como complemento, investigacio-,

.25 Popper es un precursor en este sentido pero de todos modos pensamos que es la obra de Kuhn que intenta poner la cuestión en el centro de la problemática epistemológica; en ese sentido "abre camino". Popper la trata como una entre otras importantes. 
nes empíricas, similares a las que aludíamos a propósito del tema de la política científica, cosa que no siempre aparece clara.

El fenómeno Kuhn túvo simultáneamente un paralelo en los trabajos sobre el tema de la reducción de teorías, dentro de la filosofía ortodoxa de las ciencias. La estructura de la ciencia (entre otros trabajos) de Nagel (pienso también en un trabajo de Putnam y Oppenheim en los Minnessota Studies) ya aportaba la consideración de ciertos aspectos de una "dinámica" de las ciencias. ${ }^{26} \mathrm{Y}$ no son los únicos ejemplos que vienen al caso. En este sentido el tema de la reducción de teorías (y de ciencias enteras) en Nagel significa un cambio desde dentro de la filosofía más o menos ortodoxa de la ciencia para abarcar aspectos diacrónicos a través de técnicas estáticas (en que se comparan cortes transversos de disciplinas para reconstruir la reducción).

Vale la pena tener en cuenta también las obras de Popper, Lakatos y Agassi (vínculo cierto, entre la etapa anterior, ortodoxa, y la nueva más abarcante, y además contribuciones - algunás tempranas - a ésta), con sus vueltas de tuerca en el tema, a través de sucesivas modificaciones de la tesis falsificacionista. Por otra parte las tesis de Kuhn experimentaron un tratamiento de interés en R. Suszko que nos presenta (1968) un modelo lógico-formal del desarrollo del conocimiento (como sucesión de estados) que tiene su interés aunque en un plano todavía muy abstracto. Más recientemente L: Nowak (Philosophy of Science, 1975) ha hecho un intento similar basado en los conceptos de verdad absoluta y relativa (muy lejos por tanto de la conceptuación original de Kuhn). Y aún se ha intentado (J. Kmita, Poznán Studies, 1975), inquirir cuáles son las condiciones del surgimiento y abandono de los paradigmas desde un punto de vista marxista, contradictorio por cierto del de Kuhn. Todo esto refleja una vitalidad significativa de la problemática.

Lo que nos interesa aquí, pues, es apuntar que deśde dentro de la propia filosofía de la ciencia (aun en sus versiones medianamente ortodoxas; mucho más en otras): se vio la necesidad de incluir aspectos de la producción de conocimientos, que aparecían como excluídos e inincluíbles (concepción de los dos contextos), 'en el centro mismo de su tarea.

e. De este modo tanto la dominancia de la política científica en la investigación (hipótesis 3), como la presencia inevitablè de instancias decisionales $p_{1}$, y de presupuestos, $p_{0}$, no eliminables, en "la construcción de los sistemas científicos - -que detêrminan por lo menos parcialmente el curso de la investigación-, como la inexistencia de una reconstrucción racional adecuada, si-

26 Para la discusión de la doctrina de Kuhn y problemas conexos es importante Lakatos, I. y Musgrave, G. Criticism, and the Growth of Knowledge, Cambridge, University Press, 1970. Para la teoria ortodoxa en sus nuevos desarrollos ver W. Siemens, "A Logical Empiricist Theory of Scientific Change", PSA 1970, ed." por R. Buck y R. Cohen, Dordrecht, Reidel, 1971.

26 Son sintomáticos los conceptos que incluye y sobre todo los que excluye. 
quiera reducida (con problemas complejos a todos los niveles de los enunciados de las teorías y respecto de estas mismas, en cuanto a su validación), como el desplazamiento de la filosofía de la ciencia de los problemas de estructura de los sistemas hacia los de estructura de su desarrollo, son todos fenómenos que determinan una forma distinta de considerar el conocimiento no enclaustrada en un contexto de validación excluyente.

Todos ellos apuntan a que el estado de las condiciones de producción de conocimiento acentuadas en forma creciente como determinantes, no es irrelevante para el tema de la validación. Estamos con esto muy lejos de la "falacia genética" de que nos ocuparemos seguidamente, y de toda visión absolutista de la validación. A esto ha contribuido - no podía dejar.de hacerlo- el propio proceso del conocimiento científico en nuestros días.

\section{VI}

W. Salmon, en su conocido texto de lógica (Logic, 1a ed. 1963; $2^{\text {a }}$ ed. 1973), se mantiene recalcitrante en su concepción de los dos contextos. No se trata para nada de una excepción; su exposición es ejemplar de toda una línea de pensamiento aunque posee detalles diferenciales que nos parecen poco importantes. Parte de una distinción entre inferencias y argumentos; las primeras pertenecerian al contexto de descubrimiento y las segundas al de validación. Sus ejemplos, como en la mayoría de los expositores de su posición, son basstante simples y perteneçen a la esfera psicológica o vagamente social del descubrimiento y sus efectos (la diosa Namakkal en sueños le proveyó a Ramanujan, distinguido matemático hindú, fórmulas que luego éste verificó; la manzana de Newton y sus alusiones; el hecho de que los nazis condenaran la teoría de la relatividad porque Einstein era judío; la teoría de Mendel rechazable por haber surgido en la mente de un monje; las explicaciones psicoanalíticas de lá filosofía de Platón; el argumento negativo con base concensual que sostiene: "dado que los comunistas, chinos tienden a que las mujeres casadas usen sus apellidos de solteras, es necesario que a las nuestras se les obligue a adoptar el apellido de su marido"). ${ }^{27}$ Son todos ejemplos demasiado simples, simplistas respecto a la concepción que intentan apoyar, más allá de las enormidades que señalan. Perfectamente podemos estar de acuerdo en que el sentido de estos ejemplos dados por Salmon apunta hacia la validación como posible instancia independiente, pero esto no lo exime de presentar una reconstrucción de la misma, con las dificultadeș estructurales que ello posee.

Vayamos a su argumentación. Frente "a sù acusación de falacia genética que no discutimos para esos casos -de todos modos presentados muy some${ }^{27} \mathrm{El}$ argumento aqui es que debe rechazarse -como ad hominis- una conclusion si es aceptada por un grupo que está desprestigiado. 
ramente-, parece darse en él una simétrica falacia anti-genética. Lo esencial es como sigue: las inferencias —aconteceres psicológicos_ deben ser complementadas por argumentos que están sujetos a pruebas de corrección lógica (justamente de aquí emana el prestigio del contexto de validación). No se trata sólo de la aceptación ${ }^{28}$ de enunciados sino de qué enunciados deberiamos aceptar; lo que esté determinado lógicamente. Si bien ninguna lógica permite decirnos qué inferencias hacer, sí nos permitiría, según Salmon, evaluar sus resultados: los argumentos. Debe haber una conexión lógica entre elementos de prueba y enunciados a aceptar. Sin embargo si esto fuera aś, el contexto de validación sería muy pobre: En efecto: es difícil ver - y más aún después de toda la argumentación presentada en las secciones anteriores- cómo Salmon puede pretender la existencia de tal tipo de conexión en forma general en las ciencias fácticas. Entre elementos particulares de prueba e hipótesis universales, por ejemplo, no se pueden establecer argumentos concluyentes - en el sentido indicado. Si se argumentara que Salmon no puede incurrir en error de tamaña magnitud, de todos modos no se ve bien qué otra cosa puede ser compatible con el texto; efectuándose reiteradamente un deslizamiento entre idea de validación y presencia efectiva de un concepto teóricamente explicativo. Y de modo similar con muchos otros casos de elementos de prueba y de enunciados a. aceptar: "Si usted espera que un martillo", nos dice, "haga la tarea de un desarmador usted se desilusionará pero si usted entiende su función podrá ver para qué sirve. La lógica trabaja con la justificación [validación], no con el descubrimiento, y provee herramientras para el análisis del discurso"' (p. 13). Parecería que Salmon no tiene claro para qué sirven, limitadamente, su martillo y su desarmador.

Más allá del hecho indudable de que la lógica es útil para ese fin (análisis del discurso); toda la literatura a que nos hemos referido lo atestigua pero pone de relieve a la vez, por exclusión, lo que no está sujeto a decisiones lógicas (argumentos no concluyentes); mucho más, y nada probado es pensar que se pueda dar de ese modo una reconstrucción lógica de la validación. Salmon puede contribuir, es cierto, con elementos interesantes pero no probar lo que pretende.

Terminemos de considerar su aporte con uno de esos elementos. Nos dice: "Ciertos items del contexto de descubrimiento pueden a veces ser incorporados corręctamente en el contexto de justificación mostrando que hay una conexión objetiva entre ese aspecto del descubrimiento y la verdad o falsedad de la conclusión. El argumento requiere entonces una premisa que enuncie esa conexión óbjetiva. La falacia genética consiste en citar un rasgo del descubrimiento sin proveer tal conexión con su justificación" (pp. 11-12).

28 Por un momento Salmon habla de la "aceptación" pero luego da un paso más hacia la aceptación justificada. 
Esto apunta a mostrar el carácter relevante de la validación pero de todos modos no exime a la pretendida decisión:lógica de sus carencias insalvables.

\section{VII}

No vamos a considerar todas las "consecuencias" a que dio lugar la concepción de los dos' contextos sino referirnos, apenas muy brevemente, a dos aspectos vinculados entre sí:

$i$. Pretendió usarse la distinción como fundamento, en sentido estricto, para una separación neta entre ciencia e ideología; la validación, indepen: diente, de las teorías reserva en ese caso a la ideología, entre otros factores, un papel muý secundario, una función en el descubrimiento. Sin perjuicio de poder aceptar otros argumentos que mantengan una separación-significativa entre ciencia e ideología - -pero que püeden carecer de un efecto similar al señalado-, parece indudable que sin una reconstrucción racional adecuada, sea ampliada o reducida, pero complementable, sin una elucidación y aplicación estrictas del concepto de validación, elaborado convenientemente, la "separación de contextos": no funda para nada esa demarcación entre ciencia e ideología.

ii. La independencia del marco de validación actuó de hecho, además que "de derecho", para delimitar el campo de acción profesional de la filo sofía de la ciencia (hipótesis 10), atribuyendo pues irrelevancia a un conjunto de factores que hemos señalado como parcialmente determinantes de la producción científica. De este modo - y no sólo por sus efectos profesionalesla distinción en dos contextos cumplió una función ideológica, de ocultamiento de factores relevantes, de archivo de los mismos en disciplinas (o proto-disciplinas) que de hecho no existian o apenas se iniciaban, reivindicándose para ellos su dificultad o aún:imposibilidad de someterlos a leyes, mezclándolos además con elementos que a parentemente sí poseen esas características, hundiéndolos en fin en una noche en que todas las vacas son grises. La función ideológica de la distinción tal como operó realmente en la filosofía de la ciencia durante decenios contribuyó a permitir evitar pues la consideración pública especializada de factores de creciente influencia en el desarrollo científico en cuanto a su volumen y en cuanto a su. determinación del mismo. Por ello esa función ideológica, con su dominancia, no fue para nada secundaria sino, al contrario, principal. Bastaría esto para justificar un tratamiento de esta distinción. A la pregunta de ta qué tanto discutir esta concepción de los dos contextos?, se respondería: por su dominancia; por su función ideológica, por su falta de fundamento. 


\section{VIII}

Mientras no exista una reconstrucción racional adecuada (y este "mientras" puede prolongarse bastante) se podría preguntar cuál es la salida que reconozca a la vez los factores que intervienen en la producción de conocimiento y el hechó de la validación todavía insatisfactoriamente explicada pero indudablemente actuante en la práctica científica, y que explicite el equilibrio existente entre los factores propiamente cognoscitivos y los momentos de decisión como para, de cualquier manera, poder decir que el conocimiento cientffico refleja progresivamente el mundo y permite transformarlo, circunstancias - más que virtualidades - a todas luces existentes. Con todo la práctica cientifica de cada ciencia es a nuestro entender insuficiente como lugar teórico de la explicación del concepto de validación (hipótesis 11).

No vamos a proponer aquí un ersatz en una cuestión de tremenda dificultad y complejidad (entrelazamiento de problemas). Sólo vamos a enumerar, a vía de ejemplo, algunas salidas posibles que merecen ser exploradas $\rightarrow$ o refutadas de una buena vez-, y que de hecho aparecen (algunas profusamente) en la literatura:

1. La solución kuhniana (y soluciones emparentadas) en base a comunidades de investigadores como auditorio y juez únicos del conocimiento cientifico.

2. La solución althusseriana (y soluciones emparentadas) en base a la infalibilidad de las prácticas cientificas (incluyendo a las que se autodefinen así cabiendo dudas al respecto), y en base a la inmanencia de los criterios de validación en cada ciencia, por otra parte sin especificar su, en este caso, necesario detalle. .

Hemos criticado estos tipos de soluciones, especialmente con relación a las ciencias sociales, pero con alcance general, en un trabajo sobre tres inmanentismos convergentes. ${ }^{29}$

3. Considerar que los determinantes de la producción de conocimiento y en especial los momentos decisionales son a tal punto decisivos, casi - excluyentes, como para sostener alguna variante de convencionalismo y/o en el fondo un relativismo sin fronteras. Hemos criticado una versión aparentemente atenuada en "Dos décadas de filosofía de la ciencia". 30

4. Sostener que la de validación es una idea regulativa que de todos modos permite enfocar claramente la cientificidad del conocimiento.

5. Utilización del concepto de práctica en un sentido ni inmanentista ni platonizante. Ha de reconocerse sin embargo que en la mayoria de los casos esto se ha "hecho" a través de tres o cuatro frases repetidas hasta el cansancio que pretenden resolver de modo sumario complejos problemas, sin 
que el concepto de práctica aparezca - sea como indefinido o como definidosituado dentro de una teoría y por ello sin que posea ningún carácter explicativo mínimamente discernible. Todo hace pensar que este concepto podria permitir elucidar por lo menos el proceso de justificación de decisiones.

6. Trabajar en base a ideás como las que ha propuesto Bunge de verdad parcial. $^{31}$

7. Una combinación de 5 , reelaborada, y de 6 .

\section{IX}

Dos palabras sobre nuestro modo de proceder al discutir el tema:

$i$. Se enunció un conjunto de hipótesis que están sujetas sea a comprobación empírica separada, sea a una argumentación complementaria; sólo en el caso de que estas tareas sean "cumplidas adecuadamente, pueden considerarse aquellas hipótesis como algo más que lo que corrientemente recibe el nombre de hipótesis de trabajo. Aúnque interese su plaúsibilidad, ella es totálmente insuficiente. Sin embárgó, lä los dos contextos' en su forma actual (y no está a la vista otra), no recae sobre tódas ellas sino à lo más sobre algunàs; el resto sólo concurre en el mismo sentido. En particular, la consideración de Salmon (Logic) partió solaménté dë un punto indudable: el carácter no concluyente de un conjunto significativamenté amplio de argumentos dé prueba que pretende constituir la instancia lógica de validación sin lograrlo.

ii. Sin embargo consideramos de todos modos relevantes aunque sujetas a prueba, aquellas hipótesis que apuntan a estudios empíricos de relativo detalle, por ejemplo el caso de la intervención de la política cientffica como determinante en el estado actual del desarrollo científico. Con todo pueden parecer en relación con ello, poco usuales esos enunciados de base empírica como elementos a integrar a una filosofía de la ciencia (hipótesis 12) que en su firme tradición contemporánea apareció primeramente como lógica de la ciencia y de todos modos como filosofía "dura" ("sólida"). Puede caber duda de que esas investigaciones, que incluyen elementos empíricós de tal tipö, puedan seguir llevando ése nombre (filosofía de la ciencia) -en todo caso podrían recibir otros que han sido propuestos (estudios metacientíficos, ciencias de la ciencia, etc.-, pero no se puede negar que de todos modos recogen su espiritu, y su "solidez",

iii. Podría reconstruirse formalmente, sobre la base de nuestras hipótesis

31 Presentada en su Treatise on Basic Philosophy, v. 2.

32 Después de terminado el presente trabajo püdimos constatar que R. Giere ("The Structure, Growth and Application of Scientific Knowledge", PSA 1970, p. 589-51) adopta una visión de la epistemología que es convergente con la aqul supuesta; en aspectos significativos. 
y con ayuda de algunas complementarias, a través de algunos - pocos - pasos, nuestro curso de pensamiento, lo que no nos interesa hacer aquí.

De todos modos, en to esencial, las conclusiones que han surgido son, resumidas ahora:

a. la relevancia de las condiciones de producción del conocimiento para el estudio de su validación,

b. Ia insuficiencia del concepto corrientemente utilizado de validación en la concepción de los dos contextos, y el cierre por el momento de su horizonte de utilización explicativa.

c. en todo caso se constata que el uso persistente de la distinción criticada, al pretender ocultar desde una dimensión erudita -la filosofía de la ciencia- los aspectos significativos de la producción de conocimiento y sus condiciones, que son públicamente muy importantes, revela su funcionalidad justamente ideológica; además de la ilicitud de su uso como marco rígido de la propia filosofia de la ciencia,

d. el interés que posee de todos módos la idea de validación, pero su uso teórico sujeto a reconstrucción '(cuýas elaboración y resultados resultarian previos),

e. en la medida en que se produzca una epistemología que cumpla esa tarea, se podrá determinar si se podrá hablar estrictamente de dos contextos, de producción y de validación, éste relativamente autónomo, o no. Si no se construye efectivamente una salida, no se podrá pues afirmar lícitamente la tajancia de la separación entre dos contextos ni cualesquiera consecuencias.

INSTituto DE INVESTIGaciones FiĹosóficas

Mario H. Otero Universidad Nacional Autónoma de MÉxico 